\title{
Aspects of the psychological status of patients treated with cardiac pacemakers
}

\author{
A. H. CRISP \\ M.D., M.R.C.P.(Ed.), D.P.M. \\ Professor of Psychiatry
}

\author{
E. STONEHILL \\ M.B., B.S., D.P.M. \\ Research Assistant and \\ Honorary Senior Registrar
}

Department of Psychiatry, St George's Hospital, Medical School, London, S.W.1

\begin{abstract}
Summary
An initial clinical observation that patients with externally located cardiac pacemakers are more distressed and depressed than those with internally sited pacemakers has been confirmed. The total patient group is also characterized by its tendency to report that it does not worry about getting an incurable illness. At the same time, it is evident to others that it does have such an illness. Those patients who acknowledge that they did worry in this way were found to score significantly higher on several scales of a standardized psychoneurotic index than did the overall 'pacemaker' population.

Possible reasons for the greater psychological distress displayed by the group of patients with external pacemakers are discussed.
\end{abstract}

\section{Introduction}

The Cardiac Department of St George's Hospital has, for a number of years, been concerned with the treatment of patients with heart block by cardiac pacemakers (Harris et al., 1965). This treatment is usually instigated for Stokes-Adams attacks with or without cardiac failure, if more conservative medical measures have failed. Several hundreds of patients presenting with heart block, often associated with fibrosis of the conducting tissue (Davies, 1967) but sometimes associated with more generalized myocardial damage and also with angina, have been treated in this way. The mortality rate associated with heart block has been reduced by treatment with cardiac pacemakers but is still in the order of $10 \%$ in the 1st year (Sowton, 1967) and 68\% in 5 years (Sutton et al., 1968) compared with $40-50 \%$ in the 1 st year in unpaced patients (Siddons \& Sowton, 1967).

Pacemakers are placed either externally on the body surface or internally by being implanted beneath the skin. There is an increasing tendency at St George's Hospital for pacemakers to be placed internally. Choice of site is not usually governed by the age, sex or psychological status of the patient. Pacemakers sometimes require specific siting or resiting for technical or medical reasons or because of local surgical complication. At times of resiting the new pacemaker is sometimes placed externally instead of internally or vice versa.

The physician and surgeon responsible for these patients noticed that those with externally sited pacemakers seemed more often to be depressed and worried about themselves.

\section{Present study}

The present study was designed: (a) to systematically investigate the latter observations, with an hypothesis derived from it that a group of patients with externally located pacemakers would be found to be significantly more depressed and also psychiatrically disturbed in other ways than a group with internal pacemakers; and (b) to investigate some aspects of the possible basis of the observations if confirmed.

\section{Method}

\section{(1) The population}

The population studied comprised consecutive patients attending the weekly out-patient 'pacemaker' clinic in the Cardiac Department, usually as part of routine follow-up. One hundred and twenty patients were investigated in this way.

\section{(2) Tools of enquiry}

(a) Biographical and clinical enquiry and assessment: age, sex, site of pacemaker, duration of illness as reported by patient, persistence of symptoms of any kind as reported by patient, degree of 'troublesomeness' of the pacemaker as rated on a three-point scale both by the patient and the doctor, degree of pain as rated on a four-point scale by the doctor, number of pacemakers so far used, as documented. 
(b) The Middlesex Hospital Questionnaire (M.H.Q.): this is a standardized brief diagnostic psychoneurotic index. It comprises a printed questionnaire with forty-eight questions presented on two sides of a sheet of foolscap paper. The patient usually completes the questionnaire in 5-8 $\mathrm{min}$. The responses can be rapidly scored by the doctor and are designed to provide scores (0-16) on six scales of psychiatric status, namely an anxiety scale, a phobic scale, an obsessional scale, a scale scoring somatic complaints, a depression scale and an hysteria scale. These scales have been found to be valid measures of the relevant clinical states (Crown \& Crisp, 1966) and data are available in the form of scores on a number of clinical and 'normal' groups.

\section{(3) Technique of enquiry}

(a) Biographical and clinical data were obtained and recorded by the physicians and surgeons as they saw patients in the clinic. The M.H.Q. was administered by one of us (E.S.) who was in attendance at the clinic. The aim was firstly to compare the M.H.Q. scores on the pacemaker population with data available on other populations; secondly to compare those patients with externally sited pacemakers ('external' group) with the patients with internally sited pacemakers ('internal' group).

(b) Patients for whom the pacemaker was changed from being external to being internal or vice versa. Such changes were carried out for technical or medical reasons or for reasons of local surgical complication and not on the grounds of the patient's psychiatric status. By retesting these patients after such changes, a small group of patients emerged in which each individual patient operated as his own control for the purposes of examining psychological status in relation to external and internal siting.

\section{Results}

Study 1

Table 1 displays the biographical and clinical data available on the patients.

TABLE 1. Biographical and clinical data on 120 pacemaker patients

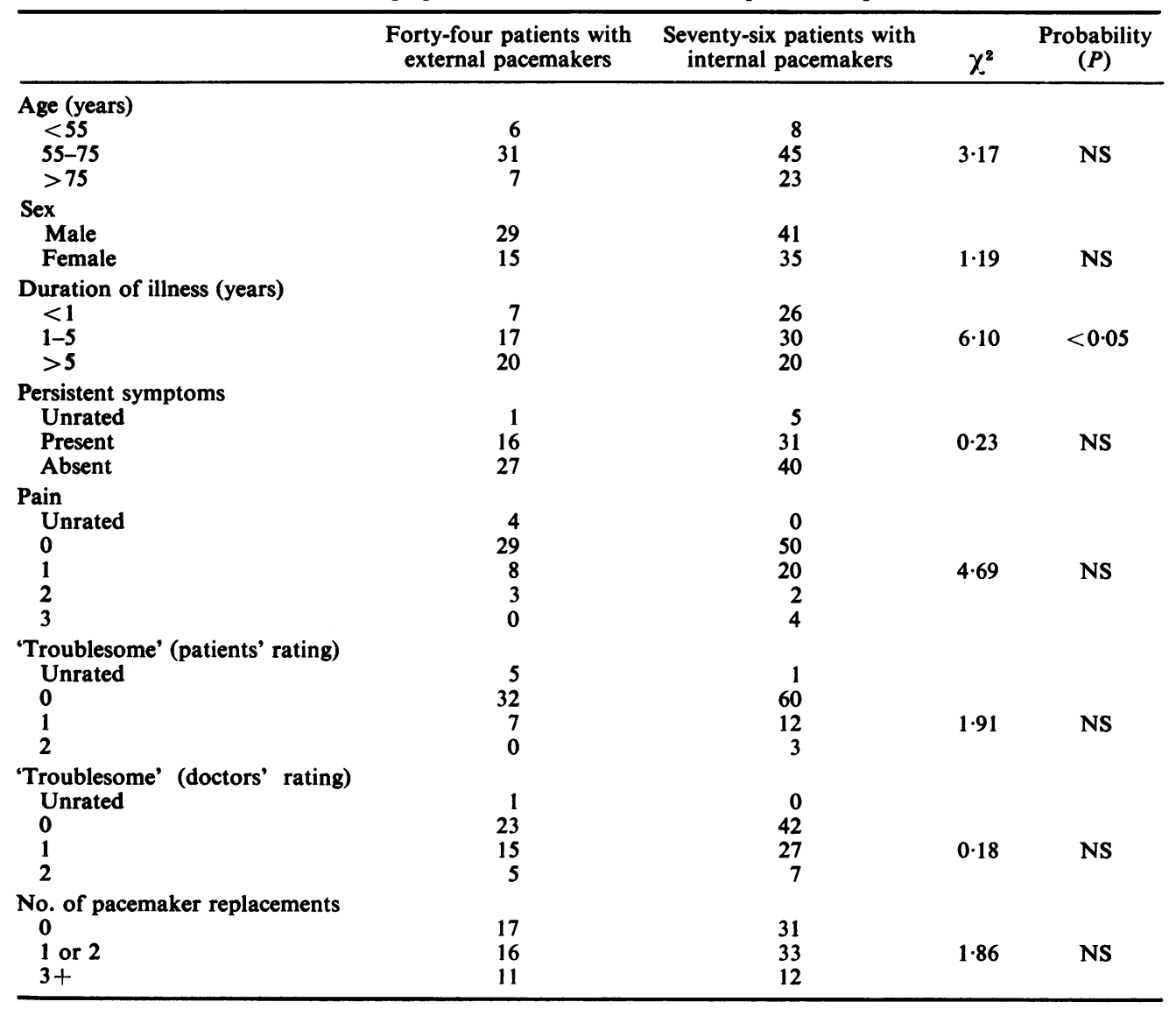


TABLE 2. Mean M.H.Q. scores and standard deviations of total group of pacemaker patients and other populations available for comparison

\begin{tabular}{|c|c|c|c|c|c|c|}
\hline & A & $\mathbf{P}$ & $\mathbf{O}$ & $\mathbf{S}$ & $\mathbf{D}$ & $\mathbf{H}$ \\
\hline 120 pacemaker patients aged $66 \cdot 3 \pm 9 \cdot 6$ years & $4 \cdot 7 \pm 3 \cdot 6$ & $5 \cdot 0 \pm 3 \cdot 5$ & $7 \cdot 8 \pm 3 \cdot 0$ & $6 \cdot 8 \pm 3 \cdot 2$ & $4 \cdot 4 \pm 2 \cdot 3$ & $2 \cdot 7 \pm 2 \cdot 5$ \\
\hline $\begin{array}{l}\text { Sixty-two consecutive psychiatric out-patients aged } \\
18-64 \text { years }\end{array}$ & $8 \cdot 4 \pm 4 \cdot 4$ & $5 \cdot 2 \pm 3 \cdot 6$ & $8 \cdot 5 \pm 3 \cdot 0$ & $7 \cdot 3 \pm 3 \cdot 3$ & $6 \cdot 3 \pm 3 \cdot 5$ & $5 \cdot 1 \pm 3 \cdot 7$ \\
\hline $\begin{array}{l}109 \text { medical students; nurses; physiotherapists aged } \\
18-22 \text { years }\end{array}$ & $5 \cdot 1 \pm 3 \cdot 1$ & $2 \cdot 9 \pm 2 \cdot 2$ & $5 \cdot 8 \pm 3 \cdot 1$ & $3 \cdot 2 \pm 2 \cdot 4$ & $3 \cdot 3 \pm 2 \cdot 3$ & $7 \cdot 5 \pm 3 \cdot 1$ \\
\hline Forty-nine midwives aged $40 \cdot 0 \pm 9 \cdot 3$ years & $5 \cdot 2 \pm 3 \cdot 5$ & $3 \cdot 7 \pm 2 \cdot 3$ & $6 \cdot 4 \pm 2 \cdot 7$ & $4 \cdot 2 \pm 2 \cdot 7$ & $3 \cdot 8 \pm 2 \cdot 8$ & $3 \cdot 9 \pm 3 \cdot 0$ \\
\hline 596 male employees at Harwell aged 40-55 years & $3 \cdot 1 \pm 2 \cdot 6$ & $2 \cdot 3 \pm 2 \cdot 1$ & $6 \cdot 7 \pm 3 \cdot 0$ & $38 \pm 2 \cdot 7$ & $2 \cdot 5 \pm 2 \cdot 4$ & $2 \cdot 8 \pm 2 \cdot 6$ \\
\hline
\end{tabular}

A, Anxiety; P, phobic; O, obsessional; S, somatic; D, depression; H, hysteria.

TABLE 3. Mean M.H.Q. scores, standard deviations and significance of differences between thirteen pacemaker patients aged 55 years or less and thirty pacemaker patients aged 75 years or more

\begin{tabular}{|c|c|c|c|c|c|c|}
\hline & A & $\mathbf{P}$ & $\mathbf{O}$ & $\mathbf{S}$ & D & $\mathbf{H}$ \\
\hline Thirteen patients aged 55 years or less & $7 \cdot 2 \pm 3 \cdot 6$ & $8 \cdot 4 \pm 4 \cdot 5$ & $9 \cdot 4 \pm 3 \cdot 5$ & $6 \cdot 6 \pm 3 \cdot 5$ & $6 \cdot 3 \pm 2 \cdot 8$ & $3 \cdot 2 \pm 2 \cdot 9$ \\
\hline Thirty patients aged 75 years or more & $4 \cdot 7 \pm 3 \cdot 6$ & $5 \cdot 2 \pm 2 \cdot 6$ & $7 \cdot 5 \pm 2 \cdot 5$ & $7 \cdot 6 \pm 3 \cdot 3$ & $4 \cdot 6 \pm 2 \cdot 2$ & $2 \cdot 2 \pm 2 \cdot 9$ \\
\hline Probability $(P)$ & $<0.05$ & $<0.01$ & NS & NS & $<0.001$ & NS \\
\hline
\end{tabular}

NS, Not significant.

TABLE 4. Mean M.H.Q. scores, standard deviations and significance of differences between forty-four external pacemaker patients and seventy-six internal pacemaker patients

\begin{tabular}{|c|c|c|c|c|c|c|}
\hline & A & $\mathbf{P}$ & $\mathbf{O}$ & $\mathbf{S}$ & $\mathbf{D}$ & $\mathbf{H}$ \\
\hline Forty-four external pacemaker patients & $4 \cdot 5 \pm 3 \cdot 9$ & $4.9 \pm 3.9$ & $8 \cdot 0 \pm 3 \cdot 2$ & $7 \cdot 0 \pm 3 \cdot 6$ & $4 \cdot 9 \pm 2 \cdot 4$ & $2 \cdot 9 \pm 2 \cdot 7$ \\
\hline Seventy-six internal pacemaker patients & $4 \cdot 9 \pm 3 \cdot 3$ & $5 \cdot 0 \pm 3 \cdot 3$ & $7 \cdot 6 \pm 2 \cdot 8$ & $6 \cdot 7 \pm 3 \cdot 0$ & $4 \cdot 0 \pm 2 \cdot 2$ & $2 \cdot 5 \pm 2 \cdot 5$ \\
\hline Probability $(P)$ & NS & NS & NS & NS & $<0.05$ & NS \\
\hline
\end{tabular}

NS, Not significant.

Groups of 'external' and 'internal' patients are not significantly different in these respects except that 'external' patients are characterized by significantly longer illness $(P<0.05)$. It is noteworthy that the patients rated 'troublesomeness' of their pacemakers significantly less highly $\left(\chi^{2}=16.85, P<0.001\right)$ than did the doctors.

The means and standard deviations of the M.H.Q. scores of the total group of pacemaker patients are displayed in Table 2 alongside M.H.Q. scores on other populations available for purposes of comparison.

The pacemaker group scores consistently lower on all scales than the psychiatric out-patient population, but scores consistently higher than all other groups on the phobic, obsessional, somatic and depression scales. For instance, they score significantly higher on the anxiety $(P<0.001)$, phobic $(P<0.001)$, somatic $(P<0.001)$ and depression $(P<0.001)$ scales than the group of 'normal' males aged 40-55. This is probably the most comparable available 'normal' group (Crown, Duncan \& Howell, 1969) to the present population.

When the mean scores of the thirty patients aged 75 years or more are compared with the mean scores of the thirteen patients aged 55 years or less (see Table 3) then the patients aged 55 years or less are found to score significantly higher on the anxiety $(P<0.05)$, phobic $(P<0.01)$ and depression $(P<$ 0.001 ) scales than the patients aged 75 years or more.

When the group of seventy-six 'internal' patients is compared with the group of forty-four 'external' patients in terms of the M.H.Q. scores (see Table 4) then the latter group is found to be characterized by significantly higher scores on the depression scale $(P<0.05)$. Duration of illness, which was a factor distinguishing between the two groups, was found not to be a major factor accounting for this significant difference of ' $D$ ' scores. However, the next study, using the patient as his own control, ensures a more direct examination of the relationship between pacemaker site and psychiatric status.

\section{Study 2}

During the study six patients had the site of their pacemaker changed from external to internal or vice versa, having already completed the M.H.Q. Four patients had the pacemaker implanted and two patients had the pacemaker exteriorized. Some weeks 
TABLE 5. Mean M.H.Q. scores, standard deviations and significance of differences in six patients who, having had the site of their pacemaker changed, repeated the M.H.Q. with the pacemaker in the new site

\begin{tabular}{lcccccc}
\hline & A & P & O & S & D & H \\
\hline External & $5 \cdot 7 \pm 3 \cdot 7$ & $5 \cdot 0 \pm 4 \cdot 1$ & $8 \cdot 8 \pm 4 \cdot 8$ & $8 \cdot 0 \pm 4 \cdot 0$ & $7 \cdot 2 \pm 2 \cdot 1$ & $5 \cdot 5 \pm 3 \cdot 0$ \\
Internal & $3 \cdot 5 \pm 1 \cdot 6$ & $3 \cdot 2 \pm 2 \cdot 6$ & $9 \cdot 7 \pm 2 \cdot 7$ & $6 \cdot 8 \pm 3 \cdot 2$ & $2 \cdot 7 \pm 1 \cdot 2$ & $3 \cdot 7 \pm 1 \cdot 0$ \\
Probability $(P)$ & $<0.05$ & $<0.01$ & NS & NS & $<0.001$ & NS \\
\hline
\end{tabular}

NS, Not significant.

TABLE 6. Mean M.H.Q. scores, standard deviations and significance of differences between twenty-three pacemaker patients who reported worrying about incurable illness and the total group of 120 pacemaker patients

\begin{tabular}{lcccccc}
\hline & A & P & O & S & D & H \\
\hline Twenty-three pacemaker patients who worry about & $8.1 \pm 3.7$ & $7.9 \pm 3.9$ & $9.2 \pm 3.4$ & $7.4 \pm 2.5$ & $5.9 \pm 3.0$ & $3.9 \pm 2.4$ \\
incurable illness & & & & & & \\
Total group of 120 pacemaker patients & $4.7 \pm 3.6$ & $5.0 \pm 3.5$ & $7.8 \pm 3.0$ & $6.8 \pm 3.2$ & $4.4 \pm 2.3$ & $2.7 \pm 2.5$ \\
Probability $(P)$ & $<0.001$ & $<0.001$ & NS & NS & $<0.02$ & $<0.05$ \\
\hline
\end{tabular}

NS, Not significant.

after the change, each patient was re-examined on the M.H.Q.

The mean scores and significance of difference between means (Sandler A test) for these two groups are displayed in Table 5. Thus, amongst these patients, external siting is significantly associated with higher scores on anxiety $(P<0.05)$, phobic $(P<0.01)$ and depression $(P<0.001)$ scales.

The possibility of six consecutive patients all changing by chance in this one predicted direction on their M.H.Q. 'depression' scores following these changes of site, is at the $2.5 \%$ level of probability (Wilcoxon matched pairs sign-ranked test).

\section{Study 3}

One of the questions in the M.H.Q. is 'Do you find yourself worrying about getting some incurable illness?'. This question falls within the 'phobic' scale for a psychoneurotic population. Four patients answered 'Often', nineteen patients answered 'Sometimes' and ninety-seven patients answered 'Never'. Three of the patients answering 'Often' and six answering 'Sometimes' came from the forty-four patients in the 'external' group; one answering 'Often' and thirteen answering 'Sometimes' came from the group of seventy-six 'internal' patients. The frequency of these responses is not significantly different between the groups. Patients responding 'Often' or 'Sometimes' were also not significantly different from the overall group in terms of age, sex, duration of illness, 'troublesomeness', pain and number of pacemakers fitted since treatment started.

Detailed data in terms of scores on individual questions in the M.H.Q. were also available on a group of forty-nine midwives aged $40 \pm 9 \cdot 3$ years. Eighteen of the forty-nine midwives responded to this question with 'Often' or 'Sometimes'. This tendency for a group of largely healthy and younger women to indicate that they worry about getting some incurable illness more often than the pacemaker population which is characterized by an incurable illness, is significant at the $5 \%$ level $\left(\chi^{2}=4 \cdot 62\right.$, $P<0.05$ ).

The group of twenty-three pacemaker patients tested on the one occasion who responded 'Often' or 'Sometimes' in contrast to 'Never' was compared with the total group of pacemaker patients. The mean scores are displayed in Table 6 . The patients who report worrying about incurable disease either 'Often' or 'Sometimes' are significantly more anxious $(P<0.001)$ as measured, more phobic $(P<0.001)$ as measured, more depressed $(P<0.02)$ as measured and more hysterical $(P<0.05)$ as measured than the total group of patients.

\section{Discussion}

In the present study a group of patients with cardiac pacemakers scores more highly on anxiety, phobic and depressive scales of a standardized psychoneurotic diagnostic questionnaire than do 'normal' populations on whom similar data are available. Although the total pacemaker population studied is significantly older than these 'normal' populations, it is the younger patients within the pacemaker population who contribute most to the high scores. Hinton (1963), in a study of the dying, found that psychological distress was more a feature of younger age groups.

When a group of patients with external pacemakers is compared with a group of patients with internal pacemakers it scores significantly higher on the depressive scale. This difference is not associated with age and sex factors which are similar for the two groups. Neither is it associated with differences 
in the degree of 'troublesomeness' of the treatment as rated by the patient and the number of previous fitments found necessary, nor the degree of pain as reported by the two groups, since these characteristics are similarly distributed in the two groups. It is associated with longer duration of illness which characterizes the 'external' group but this may have arisen because the technique of fitting external pacemakers preceded that of fitting internal pacemakers. Moreover, duration of illness was found to have a similar relationship to ' $D$ ' scores in both groups and was not held to be a major factor accounting for the difference in ' $D$ ' scores between the groups.

Furthermore, using individual patients as their own controls, six patients in whom the site of the pacemaker was changed either from external to internal or vice versa, scored significantly higher as a group on the anxiety, phobic and depressive scales whilst the pacemaker was in the external site. Moreover, all six patients changed in the predicted direction (i.e. recording higher scores with external siting whether pre- or post-rotation) on the depression scale.

These findings are taken to support and confirm the initial observations and hypothesis derived from them.

This finding raises further questions as to possible causes. Despite their infliction with an incurable disease, the majority of patients indicated in their questionnaire answers that they were never worried about getting an incurable illness. They displayed this tendency significantly more so than did a younger and more healthy female population. Within the patient group those who answered that they 'sometimes' or 'often' worried in this way scored significantly higher on several scales than did the total group. They were not distinguished from the other group either by their age or sex or in terms of 'troublesomeness', duration of illness, pain or number of pacemakers fitted.

Such worry is often a feature of patients who present with psychoneurotic illness and is sometimes regarded as a symptom of that illness. In the present population it is associated with a significantly greater degree of anxiety, phobic state and depression, as measured by the questionnaire. However, it may be that the present population's overall tendency to answer that they did not worry was an aspect of their need to deny to themselves and others the seriousness of their cardiac disorder. This may also partly explain the finding that patients regarded the pacemaker as being significantly less 'troublesome' than did the doctors. A greater psychiatric disability present as measured in the group who acknowledged worry about incurable illness may then have derived from this group's inability to deny such a real source of distress.

Finally, external location of a cardiac pacemaker may present greater psychological difficulties for the majority of patients than does internal siting of the pacemaker. Thus external fitment of pacemakers may create special psychological and experiential problems for the patient to do with the need to re-organize his body image, especially in terms of an external fitment controlling something as fundamental as the heart's activity. Yet again, it may be that external siting of the instrument serves as a more constant reminder of disability both to the patient and those around him even when he is not striving to exceed his physical limitations. Also external siting might provoke more real and irrational fears to do with the risks of accident to the instrument, neglect or even personal interference especially in those patients who are depressed, preoccupied or ambivalent about their existence.

It is concluded that, although there will be exceptions meriting special attention, the majority of patients having treatment with cardiac pacemakers will be less psychologically distressed when their pacemakers are sited internally.

\section{Acknowledgments}

The authors wish to express their gratitude to Dr Aubrey Leatham, Consultant Cardiologist, and to $\mathrm{Mr} \mathrm{A}$. H. M. Siddons, Consultant Thoracic Surgeon, who, having made the initial observations, invited the authors to conduct this study and who, together with their staff, ensured that all necessary facilities were available for the investigation and also assisted in it.

\section{References}

Crown, S. \& CrisP, A.H. (1966) A short clinical diagnostic self-rating scale for psychoneurotic patients. The Middlesex Hospital Questionnaire (M.H.Q.). Brit. J. Psychiat. 112, 917.

Crown, S., Duncan, K.P. \& Howell, R.W. (1969) Further evaluation of the Middlesex Hospital Questionnaire (M.H.Q.). Brit. J. Psychiat. (In press).

DAvies, M.J. (1967) A histological study of the conduction system in complete heart block. J. Path. Bact. 94, 351.

Harris, A., Bluestone, R., Busby, E., Davies, G., Leatham, A., Siddons, H. \& Sowton, E. (1965) The management of heart block. Brit. Heart J. 27, 469.

Hinton, J.M. (1963) The physical and mental distress of the dying. Quart. J. Med. N.s. 32, 1.

Siddons, H. \& Sowton, E. (1967) Cardiac Pacemakers, p. 141. Thomas, Springfield, Illinois.

Sowton, E. (1967) Cardiac pacemakers and pacing. Mod. conc. Cardiovasc. Dis. 36, 31.

Sutton, R., Davies, J.G., Leatham, A. \& Siddons, H. (1968) Five-year follow-up of patients paced for complete heart block. Presented at: Vth European Congress of Cardiology, Athens. 